\title{
Fiber-optic gyroscope for the suppression of a Faraday-effect-induced bias error
}

\author{
Aylin Yertutanol, ${ }^{1,2,3, *}$ (1) Önder AkçaAlan, ${ }^{3,4}$ Serdar ÖĞÜt, ${ }^{3,5}$ Ekmel Özbay, ${ }^{3,4,5}$ AND \\ Abdullah Ceylan ${ }^{1,2,6}$ \\ ${ }^{1}$ Hacettepe University, Institute of Science, Beytepe, Ankara, Turkey \\ ${ }^{2}$ Hacettepe University, Department of Nanotechnology and Nanomedicine, Beytepe, Ankara, Turkey \\ ${ }^{3}$ Bilkent University, Nanotechnology Research Center-NANOTAM, Ankara, Turkey \\ ${ }^{4}$ Bilkent University, Department of Physics, Ankara, Turkey \\ ${ }^{5}$ Bilkent University, Department of Electrical and Electronics Engineering, Ankara, Turkey \\ ${ }^{6}$ Hacettepe University, Department of Physics Engineering, Beytepe, Ankara, Turkey \\ *Corresponding author: karagoz@bilkent.edu.tr
}

Received 9 July 2021; revised 6 August 2021; accepted 6 August 2021; posted 9 August 2021 (Doc. ID 436195); published 30 August 2021

Inertial rotation sensors, interferometric fiber-optic gyroscopes (IFOGs), are widely used in military and industrial applications due to their high sensitivity and stability. In this Letter, a new, to the best of our knowledge, fiber coil design is proposed to reduce magnetic field sensitivity without adding any optical components or electronic algorithms to the IFOG system. It is shown that this design can be applied without disturbing the simplest IFOG structure. Considering the fact that the magnetic field has an invertible effect on polarization, the compensation of the Faraday-effect-induced bias error has been demonstrated theoretically and experimentally by allowing two different polarizations to travel inside the fiber coil. According to the experimental results, the bias error was reduced approximately 20 times from $\pm 9.6^{\circ} / \mathrm{h} / \mathrm{mT}$ to $\pm 0.5^{\circ} / \mathrm{h} / \mathrm{mT}$. () 2021 Optical Society of America

https://doi.org/10.1364/OL.436195

Optical sensors, such as microelectromechanical systems (MEMS), ring laser gyroscopes (RLGs), and interferometric fiber-optic gyroscopes (IFOGs), are widely used for rotation measurement in military and civilian applications. IFOG has some advantages over RLG because of its simplicity and controllable manufacturability, no moving parts, and solid-state structure $[1,2]$. Due to these characteristics, IFOG generally has been more widely used in industrial and military fields than RLG [3].

Besides IFOGs having many advantages, the sensitivity performance of IFOGs depends on the time-dependent variations in the optical path under environmental changes, such as thermal perturbations [4], vibration [5], and Earth's magnetic field [6], which cause an unwanted bias error. Among these undesired effects, phase drift caused by a magnetic field results in a Faraday-effect-induced bias error in IFOGs [7]. This is due to the phase drift of the counterpropagating light pass-through of the fiber coil. This phase drift, which will generate in the presence of a radial magnetic field, is related to polarization nonreciprocity (PNR) errors in the fiber of a fiber coil [8]. The
Faraday effect is not the principal error source of axial magnetic field drift [9]. Generally, the drift is caused by the axial direction being unable to take the advantage of optical compensation [10]. In other words, the Faraday-effect-induced bias error is directly related to the phase drift caused by PNR errors in the fiber coil. The Faraday-effect-induced bias error of IFOG can be suppressed by using a magnetic shield case with highly permeable metal [11] and photonic crystal fiber (PCF) based fiber-optic coil [12].

In addition to the above-mentioned suppression methods, optical compensation methods are proposed for PNR error control in IFOGs. Yang et al. have shown that the two orthogonal polarizations have opposite polarities and suppress the Faraday-effect-induced phase drift effectively by using a dualpolarization IFOG with the polarization beam coupler (PBC) method [13]. Zhou et al. have shown a new optical method for suppressing the radial magnetic effect by inserting a single-mode (SM) suppressing section fiber between depolarizer and fiber coil [14]. Although these methods effectively decrease magnetic errors, using new sections that violate the minimum scheme for IFOG brings new drawbacks such as complexity in design and high cost.

In the present study, a new optical compensation method called inverse symmetric polarization IFOG (ISP-IFOG) is presented, and the proposed method keeps the simplest IFOG structure. It is shown by theoretical analysis that two orthogonal polarities caused by the Faraday effect are effectively suppressed by the proposed method, and the experimental results are in good agreement with the theoretical analysis. According to the experimental results, 20 times lower magnetic field sensitivity from $\pm 9.6^{\circ} / \mathrm{h} / \mathrm{mT}$ to $\pm 0.5^{\circ} / \mathrm{h} / \mathrm{mT}$ is obtained in the radial direction.

The minimal scheme of IFOG consists of a broadband amplified simultaneous emission (ASE) source, a coupler, a multifunctional integrated optic chip (MIOC), and a polarization maintaining fiber (PMF) coil. The ISP-IFOG used in this study is remarkably similar to the "minimal scheme" shown in Fig. 1(a). In an IFOG, light from one orthogonal polarization of 


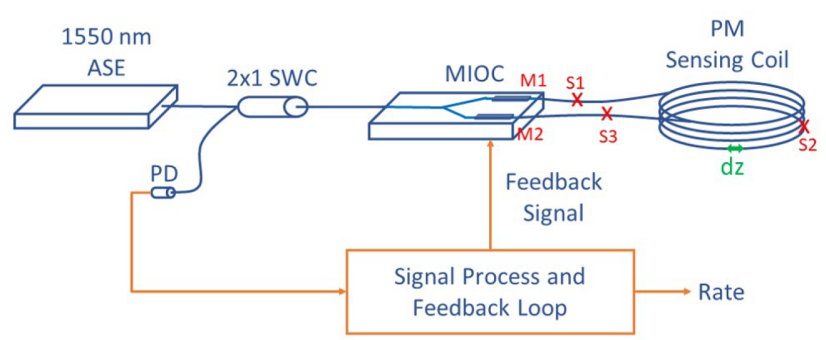

(a)

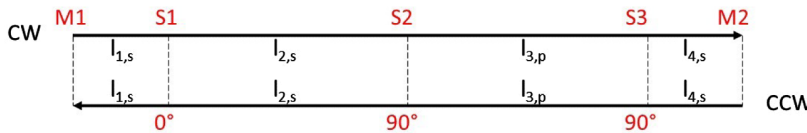

(b)

Fig. 1. Schematics of (a) detailed configuration of ISP-IFOGs and (b) CW and CCW light path.

slow and fast axes ( $s$ or $p$ polarization) propagates in a PMF coil. Unlike IFOG, in ISP-IFOG, it is aimed to have two orthogonal polarizations of light, $s$ and $p$, propagate in the PMF coil, simultaneously. M1 and M2 points in Fig. 1(a), which are the outputs of the MIOC, have $s$ polarization around $40 \mathrm{~dB}$, and $\mathrm{S} 1, \mathrm{~S} 2$, and $\mathrm{S} 3$ indicators show the splice points. By changing the splice degree of the points indicated as $\mathrm{S} 1, \mathrm{~S} 2$, and $\mathrm{S} 3$ in Fig. 1(a), the design has the potential to simultaneously provide two orthogonal polarizations of light to propagate in the PMF sensing coil.

As shown in Fig. 1(b) polarized light propagating in clockwise (CW) direction passes through $\mathrm{M} 1, \mathrm{~S} 1, \mathrm{~S} 2, \mathrm{~S} 3$, and $\mathrm{M} 1$, respectively, while light propagating in the counter-CW (CCW) direction passes through M1, S3, S2, S1 and M2, respectively. It is clearly seen in Fig. 1(b) that the MIOC pigtail fiber segments are identified as $l_{1}, l_{4}$. The fiber segments of fiber coil identified as $l_{2}, l_{3}$, which have a splice point S2 between two fiber segments, have the same length.

When splice points $S 1, S 2$, and $S 3$ are at a $0^{\circ}$ splice angle, $s$-polarization mode propagates in the sensing coil, while splice points $S 1$ and $S 3$ are at a $90^{\circ}$ and $S 2$ is at a $0^{\circ}$ splice angle; $p$-polarization mode propagates in the sensing coil as in the sensing coil of IFOG.

The bias error in IFOG can be calculated by the Jones matrix method [15]. The Faraday effect is valid only when there is a twist that is inevitable in practice during the process of drawing the fiber and winding the PMF coil [16]. Therefore, as shown in Fig. 1(a), $d z$ is the micro unit corresponding to the random twist. Then, the basic equation of the input and output electrical fields under the magnetic fields of $d z$ is given by [15]

$$
\left[\begin{array}{l}
\hat{E}_{x} \\
\hat{E}_{y}
\end{array}\right]=\exp \left(-j \beta_{a v} z\right) C(\phi, \zeta)\left[\begin{array}{l}
\hat{E}_{x} \\
\hat{E}_{y}
\end{array}\right],
$$

where $C$ can be described as a transfer matrix:

$$
C(\phi, \zeta)=F(\phi, \zeta)+G(\phi, \zeta),
$$

where

$$
F(\phi, \zeta)=\left[\begin{array}{cc}
\cos \eta z-j \sin \eta z & 0 \\
0 & \cos \eta z+j \sin \eta z
\end{array}\right],
$$

$$
\begin{gathered}
G=\left[\begin{array}{cc}
g_{1} & 1 \\
-1 & g_{1}^{*}
\end{array}\right] \cdot g_{2} \sin \eta z \\
g_{1}=j \frac{\phi-\zeta}{\eta+\Delta \beta / 2} \text { and } g_{2}=\frac{\phi-\zeta}{\eta}, \\
\eta=\sqrt{\left(\frac{\Delta \beta}{2}\right)^{2}+\left(\phi-\zeta^{2}\right)}
\end{gathered}
$$

The birefringence, twist, and Faraday rotation per unit length are represented by $\Delta \beta, \phi$, and $\zeta$, respectively.

The total transfer matrix of the fiber spool for each section can be expressed by

$S=C_{n} C_{n-1} \ldots C_{1}=\left(F_{n}+G_{n}\right)\left(F_{n-1}+G_{n-1}\right) \ldots\left(F_{1}+G_{1}\right)$.

By using $\eta_{k} z_{k}=2 \pi$, where $k=1,2, \ldots, n-1$,

$$
z_{k}=\frac{2 \pi}{\eta_{k}} \simeq 2 \frac{2 \pi}{\Delta \beta} .
$$

Thus, the transfer matrix $S$ can be written as a unit matrix as shown in Eq. (9) [15]:

$$
S=\left(F_{n}+G_{n}\right) U(n),
$$

where

$$
U(n)=\left[\begin{array}{cc}
\exp (-j \cdot 2(n-1) \pi) & 0 \\
0 & \exp (j \cdot 2(n-1) \pi)
\end{array}\right] .
$$

The transfer matrix $S$ can be written as a combination of the fiber sections between the connection points (M1, S1, S2, S3, M2) as shown in Figs. 1(a) and 1(b), and the unit matrix becomes

$$
S=S_{1} S_{2} S_{3} S_{4}
$$

Applying Eqs. (9) and (11) the transfer matrix of the light through CW and CCW directions in the fiber coil is given as

$$
\begin{aligned}
S_{c w}= & \left(F_{n_{1}}+G_{n_{1}}\right) U\left(n_{1}\right) R\left(\alpha_{1}\right)\left(F_{n_{2}}+G_{n_{2}}\right) U\left(n_{2}\right) R\left(\alpha_{2}\right) \\
& \left(F_{n_{3}}+G_{n_{3}}\right) U\left(n_{3}\right) R\left(\alpha_{3}\right)\left(F_{n_{4}}+G_{n_{4}}\right) U\left(n_{4}\right), \\
S_{c c w}= & \left(F_{n_{4}}+G_{n_{4}}\right) U\left(n_{4}\right) R\left(\alpha_{3}\right)\left(F_{n_{3}}+G_{n_{3}}\right) U\left(n_{3}\right) R\left(\alpha_{2}\right) \\
& \left(F_{n_{2}}+G_{n_{2}}\right) U\left(n_{2}\right) R\left(\alpha_{1}\right)\left(F_{n_{1}}+G_{n_{1}}\right) U\left(n_{1}\right),
\end{aligned}
$$

where

$$
\begin{gathered}
\eta_{c w}=\sqrt{\left(\frac{\Delta \beta}{2}\right)^{2}-\left(\phi_{c w}-\zeta_{c w}\right)^{2},} \\
\eta_{c c w}=\sqrt{\left(\frac{\Delta \beta}{2}\right)^{2}+\left(\phi_{c c w}+\zeta_{c c w}\right)^{2}}, \\
R(\alpha)=\left[\begin{array}{ll}
\cos \alpha-\sin \alpha \\
\sin \alpha & \cos \alpha
\end{array}\right] .
\end{gathered}
$$

$R(\alpha)$ is used to represent the degree of splice points shown in Fig. 1(b). In ISP-IFOG, the configuration $0^{\circ}-90^{\circ}-90^{\circ}$ 


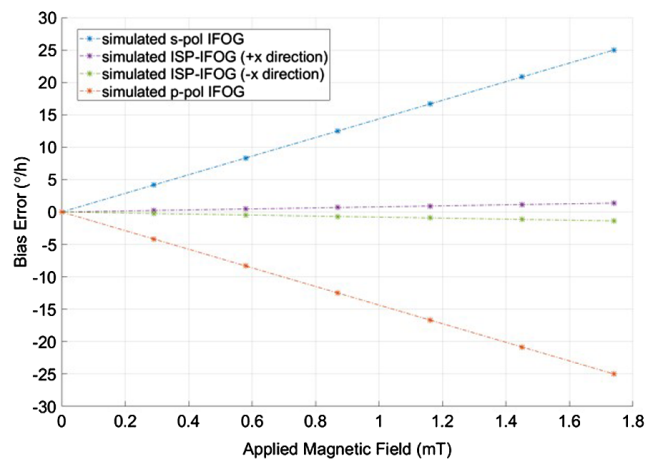

Fig. 2. Simulation results of the two orthogonal polarity axes of IFOG and ISP-IFOG recorded for the different magnetic field intensities.

angle of the splice is used for $\alpha_{1}, \alpha_{2}$, and $\alpha_{3}$, respectively. In addition to that, the polarizer element of MIOC has an infinite extinction ratio such that only $(1,1)$ elements of $S_{c w_{n}}$, $S_{c c w_{n}}$ can be considered. In addition, when $\eta \simeq \Delta \beta \gg \phi$ and $\eta \simeq \Delta \beta \gg \zeta$ are assumed, then $(1,1)$ elements of $G_{c w_{n}}$ and $G_{c c w_{n}}$ can be neglected due to the low impact of the second order [15]. Thus, the phase difference is given by Eqs. (17) and (18):

$$
\Delta \psi=\int_{0}^{l_{1}+l_{4}}\left(\eta_{c w}-\eta_{c c w}\right) \mathrm{d} z=\int_{0}^{l_{1}+l_{4}}\left(\frac{4 \phi \zeta}{\eta_{c w}+\eta_{c c w}}\right) \mathrm{d} z .
$$

$l_{1}+l_{4}$ is the total length of the MIOC pigtail fibers, and $\phi, \zeta, \eta_{c w}$, and $\eta_{c c w}$ depend on $z$. This phase difference can be expressed as $\operatorname{drift} \delta$ :

$$
\delta=\frac{8 \zeta_{0} r}{\Delta \beta} \cdot \sqrt{2 m \pi W}, \quad m=\frac{l_{1}+l_{4}}{2 \pi r} .
$$

When the fiber segments of fiber coil $l_{2}$ and $l_{3}$ have equal length, it has been shown that the derivation of phase drift is totally suppressed in the fiber coil, where $W$ is the power spectrum of the twist. Although the phase drift is dependent on both the fiber length of the fiber coil and the fiber length of the MIOC pigtail in the IFOG configuration, the Faraday-effect-induced bias error is dependent on only the fiber length of the MIOC pigtails $l_{1}$ and $l_{4}$ in the ISP-IFOG configuration. Additionally, the bias can be linearly reduced to zero, while the fiber length of the MIOC pigtails $l_{1}$ and $l_{4}$ are short enough. The Faradayeffect-induced bias error is calculated numerically by MATLAB using Eq. (18) for IFOG and ISP-IFOG with a MIOC pigtail fiber length of $1.5 \mathrm{~m}$ and fiber coil fiber length of $1100 \mathrm{~m}$ (Fig. 2). Additionally, it can be thought that these splice points' angle errors could cause additional drift. It was briefly described by Hotate and Tabe that the drift caused by the Faraday effect is not enhanced by any misalignment of the polarization axes but just the intensity of the optical power changes measured by the photodetector [15]. By using a broadband light source, the coherence length is reduced as much as possible, and interference between residual signals and main signals is prevented [17]. Also, the polarization error effects in the opposite axis are eliminated by the MIOC with a high polarization value [17].

The Faraday-effect-induced bias error of IFOG and ISPIFOG are tested and compared with each other to see the difference between experimental results and numerical calculations. As shown in Fig. 1(a), to eliminate the interference

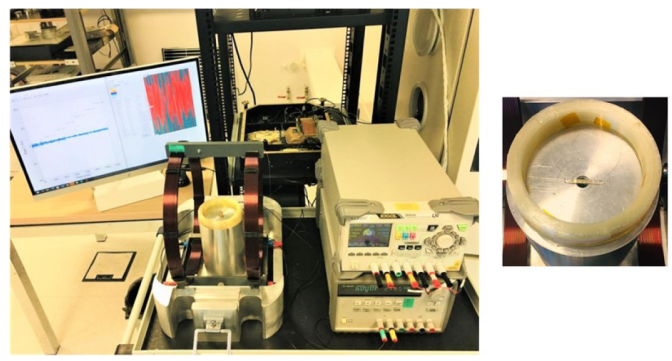

Fig. 3. Experimental measurement setup for fiber coil and MIOC under a radial magnetic field.

errors due to splice angle errors, backreflections, and poor quality of polarization maintaining optical components, a $15 \mathrm{~nm}$ broadband ASE light source with a central wavelength at $1550 \mathrm{~nm}$ is used and coupled to a $3 \mathrm{~dB}$ optical coupler. Phase modulation is given by a MIOC fabricated by the fiber-optic (APE) method that has functioned as a $>40 \mathrm{~dB}$ polarizer, a $3 \mathrm{~dB}$ coupler, and a phase modulator. PMF with $80 \mu \mathrm{m}$ cladding and $165 \mu \mathrm{m}$ coating diameter is chosen to wind around average in an $85 \mathrm{~mm}$ diameter spool to make the fiber sensing coil. For the sensing coil, $1100 \mathrm{~m}$ PMF is made up of 40 layers of fiber with a quadrupole winding pattern that is tested. A photodetector is implemented to detect the interfered signal that comes from the sensing coil. A closed-loop modulation technique is used for the rate measurement to increase the stability and linearity of the scale factor. The most important feature of ISP-IFOG that makes it more competent than IFOG is the splice point in the middle of the fiber coil that provides two orthogonal polarizations to travel into the fiber coil simultaneously.

The Helmholtz coil, which is made up of copper wire, is used to provide uniform magnetic field as shown in Fig. 3. The fiber coil and the MIOC are placed in the center of the Helmholtz coil, and the magnetic field direction is adjusted parallel to the sensitivity axis of the fiber coil. The magnitude of the magnetic field can be controlled by a DC power supply that is altered between $+1.75 \mathrm{mT}$ and $-1.75 \mathrm{mT}$.

In the experimental measurement setup for IFOG, two orthogonal polarities (slow and fast axes) of light traveling in a fiber coil are individually measured. The radial magnetic field is varied from 0 to $1.75 \mathrm{mT}$ by $0.29 \mathrm{mT}$ steps, and it is observed that the phase drift of two orthogonal polarizations gives the same magnitude bias error with different signs. Sensitivity to a temperature change called a Shupe error is eliminated by performing a measurement under constant room temperature. Before the experimental analysis of the Faraday-effect-induced bias error, it was confirmed that this configuration had a small bias error by testing the IFOG and ISP-IFOG in a static environment without an external magnetic field. It was confirmed that an additional splice point in the middle of the coil had not caused zero drift. According to the results, the biases of IFOG and ISP-IFOG are measured as $9.6(\% / \mathrm{h})$ and $9.8(\% / \mathrm{h})$, respectively, in Ankara, Turkey. Power and polarization measurements were done after each splice point in this configuration, and there was no additional splice loss. Using the measurement results of bias drift, the bias error around zero is calculated for IFOG and ISP-IFOG.

To measure Faraday-effect-induced bias error, seven measurements were made for each configuration by applying seven different magnetic fields of different intensities each with a period of $180 \mathrm{~s}$. According to the experimental results given 


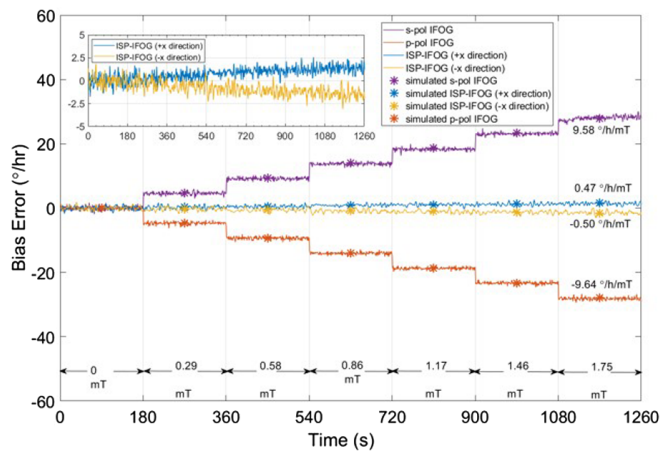

Fig. 4. Experimental and simulation results of two different polarity axes of IFOG and ISP-IFOG recorded for the different magnetic field intensities over a period of $180 \mathrm{~s}$.

in Fig. 4 , the bias error $(\% / h)$ increases linearly by the magnetic field intensity. Based on these experimental results, the bias error of IFOG is calculated as $9.58^{\circ} / \mathrm{h} / \mathrm{mT}$ and $-9.64^{\circ} / \mathrm{h} / \mathrm{mT}$ for slow axis and fast axis polarization, respectively. It should be noted that in the IFOG configuration, the magnitude of the bias error is almost the same, with different signs for two orthogonal polarizations.

The same measurement setup is used to measure the bias error of ISP-IFOG with the same magnetic field intensities. The degree of splicing angle rotates from $0^{\circ}-90^{\circ}-90^{\circ}$ for points S1, S2, S3 (Fig. 1), respectively. The bias errors of ISP-IFOG for the $+x$ and $-x$ directions of a magnetic field are $0.47^{\circ} / \mathrm{h} / \mathrm{mT}$ and $-0.50^{\circ} / \mathrm{h} / \mathrm{mT}$, respectively (Fig. 4). It is clearly seen that the bias error is significantly suppressed. The reason for the small amount of bias is the fiber length of the MIOC pigtails $l_{1}$ and $l_{4}$, which is $1.5 \mathrm{~m}$ with the same polarity axes ( $s$ polarization).

It can be clearly seen that the bias error of the gyroscope under a magnetic field can be reduced considerably with ISPIFOG design. According to Eq. (18), total phase difference is equal to the sum of the phases of the light traveling through the fiber coil in slow and fast polarization axes. Due to the fact that the slow and fast polarization axes under the magnetic field have an inverse polarization, the Faraday effect can be greatly reduced when both the slow and fast axes that travel in the fiber coil are equal. This can be achieved by using two equal lengths of the fiber segments $l_{2}$ and $l_{3}$ as shown in Fig. 1 . This is proven by theoretical analysis and verified by experimental results. According to the ISP-IFOG phase drift measurements under a magnetic field, the bias error is calculated as less than $0.50^{\circ} / \mathrm{h} / \mathrm{mT}$ of ISP-IFOG. This error can be due to the fact that both lengths of $l_{2}$ and $l_{3}$ have small differences due to practical limitations, and the lengths of $l_{1}$ and $l_{4}$ have the same polarization axes.

The ISP-IFOG configuration is tested, and it is shown that the radial magnetic field sensitivity is decreased considerably, without generating an optical or electronic algorithm in the IFOG system. Due to the invertible effect of the magnetic field on the polarization of light inside the fiber, it has been observed that the Faraday-effect-induced bias error in the IFOG system is suppressed 20 times from $\pm 9.6^{\circ} / \mathrm{h} / \mathrm{mT}$ to $\pm 0.5^{\circ} / \mathrm{h} / \mathrm{mT}$ without decreasing the sensitivity of IFOG. Thus, ISP-IFOG offers a promising way for substantial suppression of the bias error caused by the Faraday effect in an IFOG. This configuration also has a potential to decrease the sensitivity of the temperature dependence of phase drift. For future work, the detailed investigation of ISP-IFOG bias error under temperature variations is planned.

In conclusion, the Faraday-effect-induced bias error of IFOGs was analyzed in terms of the magnetic field sensitivity of the fiber coil. Numerical calculations are carried out to determine the Faraday-effect-induced bias error of an IFOG, and this error is also analyzed by experiments under different magnetic field intensities for the same fiber coil geometry. It has been confirmed that the ISP-IFOG is essential in suppressing the Faraday-effect-induced bias error over a radial magnetic field.

Funding. Türkiye Bilimler Akademisi.

Acknowledgment. This work is partially produced from the Ph.D. studies of A.Y. One of the authors (E.O.) also acknowledges partial support from the Turkish Academy of Sciences.

Disclosures. The authors declare no conflicts of interest.

Data Availability. Data underlying the results presented in this paper are not publicly available at this time but may be obtained from the authors upon reasonable request.

\section{REFERENCES}

1. S. Merlo, M. Norgia, and S. Donati, Handbook of Optical Fibre Sensing Technology (2000), pp. 1-23.

2. K. Grattan and T. Sun, Sens. Actuators A Phys. 82, 40 (2000).

3. G. A. Sanders, B. Szafraniec, R.-Y. Liu, C. L. Laskoskie, L. K. Strandjord, and G. Weed, Proc. SPIE 2837, 61 (1996).

4. X. Chen and R. Liu, Proc. SPIE 8418, 410 (2012).

5. C. Shen, X. Chen, J. Yu, and J. Wu, in IEEE International Instrumentation and Measurement Technology Conference (2011), pp. 1-5.

6. G. A. Pavlath, Proc. SPIE 2837, 46 (1996).

7. H. C. Lefevre, Proc. SPIE 2837, 2 (1996).

8. Y. Zhao, Y. Zhou, D. Zhang, J. Yang, Y. Zhou, S. Shi, X. Shu, and C. Liu, IEEE Sens. J. 15, 5128 (2015).

9. P. Liu, X. Li, X. Guang, Z. Xu, W. Ling, and H. Yang, Opt. Commun. 394, 122 (2017).

10. Y. Zhang, S. Qiao, L. Sun, Q. W. Shi, W. Huang, L. Li, and Z. Yang, Opt. Express 22, 11070 (2014).

11. K. Böhm, K. Petermann, and E. Weidel, Opt. Lett. 7, 180 (1982).

12. H. Wen, M. A. Terrel, H. K. Kim, M. J. F. Digonnet, and S. Fan, J. Lightwave Technol. 27, 3194 (2009).

13. Y. Yang, Z. Wang, and Z. Li, Opt. Lett. 37, 2841 (2012).

14. Y. Zhou, Y. Zhao, H. Tian, D. Zhang, T. Huang, L. Miao, X. Shu, S. Che, and C. Liu, Opt. Eng. 55, 126106 (2016).

15. K. Hotate and K. Tabe, Appl. Opt. 25, 1086 (1986).

16. T. Saida and K. Hotate, J. Lightwave Technol. 17, 222 (1999).

17. H. C. Lefevre, The Fiber-Optic Gyroscope (Artech House, 2014). 